\title{
Nonlinear Dynamics of Large Fluctuations
}

\author{
Mark I. Dykman*, Vadim N. Smelyanskiy*, \\ Colin J. Lambert ${ }^{\dagger}$, Dmitrii G. Luchinsky ${ }^{\dagger 1}$, \\ Peter V.E. McClintock ${ }^{\dagger}$ \\ -Department of Physics and Astronomy, Michigan State University, \\ East Lansing, MI 48823, USA \\ ${ }^{\dagger}$ School of Physics and Chemistry, Lancaster University \\ Lancaster, $L A 1 \& Y B, U K$
}

\begin{abstract}
The distribution of paths for large fluctuations away from a stable state has been investigated, theoretically and by analog experiment. We have found critical broadening of the distribution of the paths coming to a cusp point representing the simplest generic singularity in the pattern of most probable (optimal) fiuctuational paths in a non-equilibrium system. The critical behavior can be described by a Landau-type theory.
\end{abstract}

\section{INTRODUCTION}

A wide variety of physical phenomena, ranging from nucleation at phase transitions to failures of electronic devices, are driven by large rare fluctuations. In many cases the fluctuating systems of interest are far from thermal equilibrium. It was recognized by Onsager and Machlup [1] that an insight into the physics of large fluctuations can be gained from an analysis of the distribution of fuctuational paths along which the system moves to a given state. This distribution is a fundamental characteristic of the fluctuation dynamics, and its understanding paves the way to controlling the fluctuations. It peaks sharply at the optimal (i.e. most probable) path; we report below the first experimental observations of this pattern.

In thermal equilibrium, with white noise, the optimal paths are timereversed deterministic paths [2]. But this is not true for nonequilibrium systems, because they lack time reversibility. The statistical distribution of

1) Permanent address: Russian Institute for Metrological Service, Ozernaya 46, 119361 Moscow, Russia. 
nonequilibrium systems may have singular features [3], and so may the pattern of optimal paths. Optimal paths represent the extrema of a variational problem. A generic property of the pattern of extreme paths is the occurrence of caustics [4], in general starting in pairs from a cusp (focal) point: see Fig. 1.

Caustics and cusps in the pattern of extreme paths have been found numerically [5] and investigated analytically [6]. Note that contributions to the probability distribution from different paths are all positive: interference effects do not occur and the singularities in the pattern of optimal paths are therefore different from those known for wave fields [6]. Although optimal fluctuational paths do not encounter caustics, but they can focus into cusp points. Use of the prehistory probability density [7] (see below) enables us to reveal and analyze the singular behavior, theoretically and experimentally [8].

\section{SINGULAR BEHAVIOR OF THE DISTRIBUTION OF FLUCTUATIONAL PATHS}

The simplest system that displays singularities of optimal paths is an overdamped Brownian particle driven by a periodic force $K(q ; t)$,

$$
\begin{aligned}
& \dot{q}=K(q ; t)+\xi(t), \quad K(q ; t)=K(q ; t+T), \\
& \left\langle\xi(t) \xi\left(t^{\prime}\right)\right\rangle=D \delta\left(t-t^{\prime}\right) .
\end{aligned}
$$

For small noise intensities $D$, within a relaxation time $\tau_{r}$ the system (1) will approach the stable periodic state $q^{(0)}(t), \dot{q}^{(0)}=K\left(q^{(0)} ; t\right), q^{(0)}(t+T)=$ $q^{(0)}(t)$, and a periodic stationary probability distribution will be formed in the basin of attraction to this state.

Analysis of the prehistory probability density, $p_{h}\left(q, t \mid q_{f}, t_{f}\right)[7]$ enables the distribution of paths in large fluctuations to be investigated and visualized. It is the conditional probability density for a system that had been fluctuating about $q^{(0)}(t)$ for a time greatly exceeding $\tau_{r}$, and arrived to the point $q_{f}$ at the instant $t_{f}$, to have passed through the point $q$ at the instant $t\left(t<t_{f}\right)$. It can be written as a path integral:

$$
\begin{aligned}
& p_{h}\left(q, t \mid q_{f}, t_{f}\right)=\text { const } \times \int_{q\left(t_{i}\right) \approx q^{(0)}\left(t_{i}\right)}^{q\left(t_{f}\right)=q_{f}} \mathcal{D} q\left(t^{\prime}\right) \exp \left[-\frac{S[q(t)]}{D}\right] \\
& \times \delta(q(t)-q), t_{i} \rightarrow-\infty, \int d q p_{h}\left(q, t \mid q_{f}, t_{f}\right)=1
\end{aligned}
$$

Here, $S[q]$ determines the probability distribution over the paths of a Markovian system. To lowest order in the noise intensity $D$ it takes the form of the action functional for an auxiliary dynamical system with the Lagrangian $L(\dot{q}, q ; t)$ (cf. [9]):

$$
S[q(t)]=\int_{t_{i}}^{t_{f}} d t L(\dot{q}, q ; t), L(\dot{q}, q ; t)=\frac{1}{2}[\dot{q}-K(q ; t)]^{2} .
$$




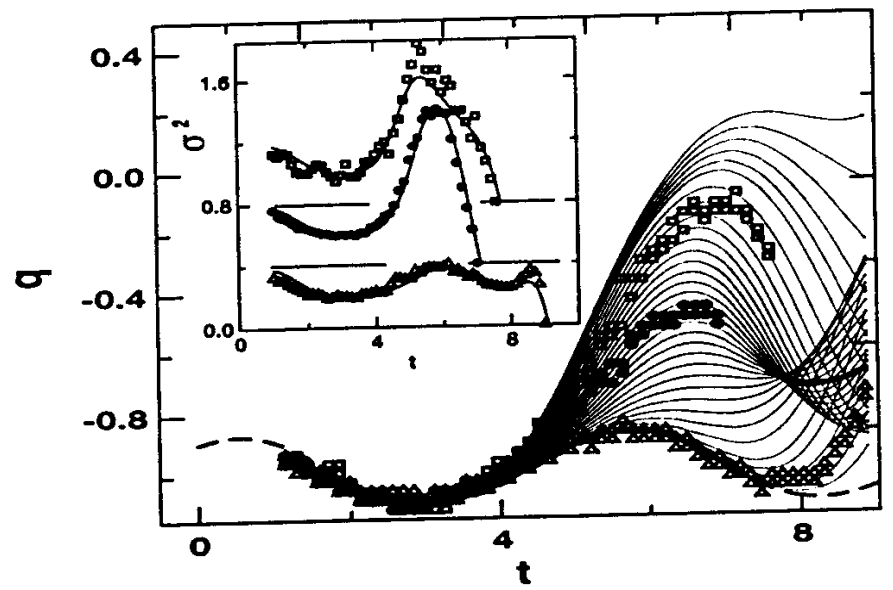

FIGURE 1. Extreme paths of (1) for $K=q-q^{3}+0.264 \cos 1.2 t$. The stable state $q^{(0)}(t)$ from the vicinity of which the paths start is shown by the dashed line. The bold line emanating from the cusp point is the switching line calculated for $D \rightarrow 0$. The data points show the maxima of the prehistory probability distribution measured for three final points away from the cusp. Reduced variances of the corresponding Gaussian distributions $\sigma^{2}$ (displaced along the ordinate axis for clarity) are compared with the theory in the inset.

The optimal (most probable) fluctuational path $q_{\mathrm{opt}}\left(t \mid q_{f}, t_{f}\right)$ along which the system arrives at $q_{f}$ at time $t_{f}$ is found by solving the variational problem

$$
\begin{aligned}
& \frac{\delta S[q]}{\delta q(t)}=0, \quad q_{\mathrm{opt}}\left(t_{f} \mid q_{f}, t_{f}\right)=q_{f}, \\
& q_{\mathrm{opt}}\left(t_{i} \mid q_{f}, t_{f}\right) \rightarrow q^{(0)}\left(t_{i}\right) \text { for } t_{i} \rightarrow-\infty .
\end{aligned}
$$

But Eq.(4) describes extreme fluctuational paths, which are not necessarily the optimal paths that provide the global minimum of the action $S[q]$ and are therefore of physical significance. Extreme paths $q(t)$ as given by (3), (4) can intersect, since a dynamical system with the Lagrangian (3) is nonintegrable. In contrast, generically only one optimal path can arrive at a given point.

The shape of the prehistory probability density $p_{h}(2)$ can be found by expanding the coordinate $q(t)$ into the orthonormal functions $\psi_{n}(t)$ which diagonalize the second variation of the action:

$$
q(t)=q_{\mathrm{opt}}\left(t \mid q_{f}, t_{f}\right)+\sum_{n} a_{n} \psi_{n}(t)
$$

It follows from (3) that the functions $\psi_{n}(t)$ satisfy a Schrödinger-type equation

$$
-\ddot{\psi}_{n}+V(t) \psi_{n}=\lambda_{n} \psi_{n}, V(t)=\left[\frac{\partial^{2} K}{\partial q \partial t}+\frac{1}{2} \frac{\partial^{2} K^{2}}{\partial q^{2}}\right]_{\mathrm{opt}}
$$




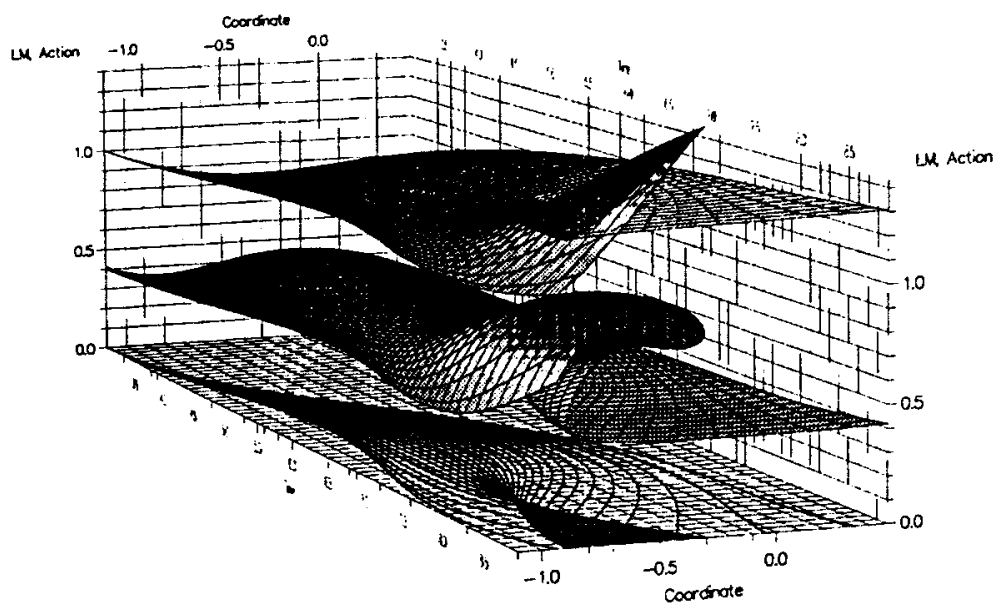

FIGURE 2. Generation of singularities: (middle plot) LM in the space $(t, q, p)$ with two folds; (lower plot) projection of LM onto $(t, q)$ plane; (upper plot) multivalued action surface $S(q, t)$.

with the boundary conditions $\psi_{n}\left(t_{i}\right)=\psi_{n}\left(t_{f}\right)=0$ (in (6) the derivatives of $K \equiv K(q ; t)$ are evaluated for $\left.q=q_{\mathrm{opt}}\left(t \mid q_{f}, t_{f}\right)\right)$.

For trajectories $q(t)$ close to the optimal path, the $a_{n}$ in (5) are small, and the action $S[q(t)]$ is quadratic in $a_{n}$ (unless $\left(q_{f}, t_{f}\right)$ is close to the cusp: see below),

$$
\begin{aligned}
& S[q(t)]=S\left(q_{f}, t_{f}\right)+s\left(\left\{a_{n}\right\}\right), s\left(\left\{a_{n}\right\}\right)=\frac{1}{2} \sum_{n} \lambda_{n} a_{n}^{2}, \\
& S\left(q_{f}, t_{f}\right) \equiv S\left[q_{\mathrm{opt}}\left(t \mid q_{f}, t_{f}\right)\right] .
\end{aligned}
$$

If one writes the path integral (2) as an integral over all $a_{n}$ and substitutes Eqs. (5), (7) into (2), one obtains

$$
\begin{aligned}
& p_{h}\left(q, t \mid q_{f}, t_{f}\right)=M \exp \left(-\frac{\left[q-q_{\mathrm{opt}}\left(t \mid q_{f}, t_{f}\right)\right]^{2}}{2 D \sigma^{2}\left(t \mid q_{f}, t_{f}\right)}\right) \\
& \sigma^{2}\left(t \mid q_{f}, t_{f}\right)=\sum_{n} \lambda_{n}^{-1} \psi_{n}^{2}(t), M=\left(2 \pi D \sigma^{2}\right)^{-1 / 2}
\end{aligned}
$$

Near the maximum, the distribution $p_{h}$ is evidently Gaussian in the distance of $(q, t)$ from the optimal path $q_{\mathrm{opt}}\left(t \mid q_{f}, t_{f}\right)$ (cf. [7]). Therefore by investigating $p_{h}$ one can find directly the optimal path itself, and also analyze the shape of the tube of paths arriving at a given point $\left(q_{f}, t_{f}\right)$. Away from the cusp point, the width of this tube is $\propto D^{1 / 2}$. The reduced width of the distribution (8) $\sigma\left(t \mid q_{f}, t_{f}\right)$ is independent of $q_{f}, t_{f}$ for $t_{f}-t \gg \tau_{r}$ : it gives the reduced 


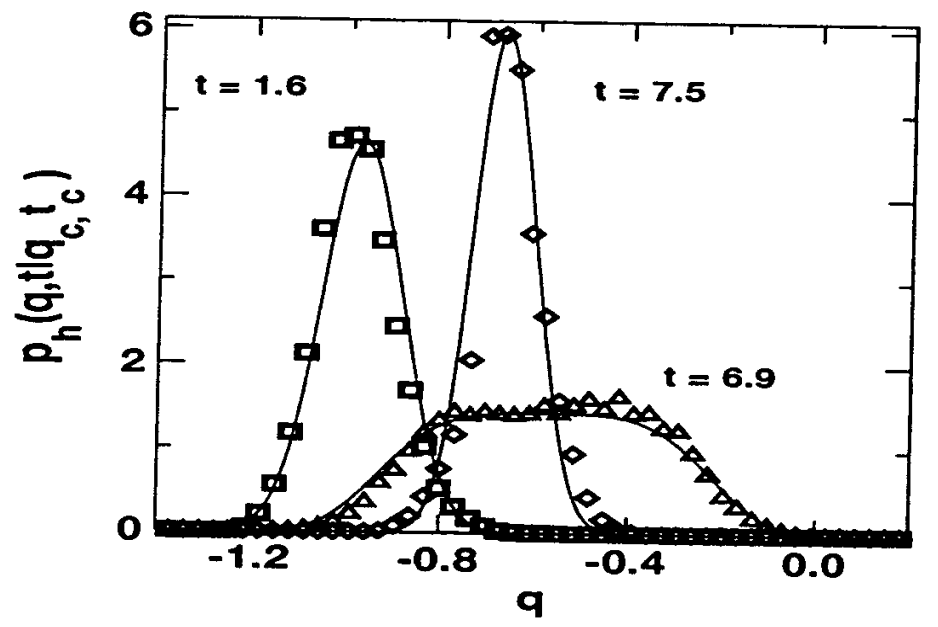

FIGURE 3. Cross-sections of the prehistory probability distribution calculated (curves) and measured (points) for fluctuations to the cusp point in Fig. $1\left(\left(q_{\mathrm{c}} \approx-0.70, t_{\mathrm{c}} \approx 7.69\right)\right.$, for three values of $t$. The distribution is Gaussian very close to, and far from, the cusp; but it is critically broadened and strongly non-Gaussian at intermediate values of $t$.

width of the stationary Gaussian distribution about $q^{(0)}(t)$. Experiments on an analog electronic model of (1), based on a standard technique [10], have yielded results which are in a very good agreement with Eqs. (4), (8); see Fig. 1 (the explicit form of $\sigma\left(t \mid q_{f}, t_{f}\right)$ is given in [8]).

Eq. (8) does not apply if the final point $\left(q_{f}, t_{f}\right)$ is close to a singularity of the pattern of extreme paths [8]. The origin of the singularities can be understood from topological arguments [6]: see Fig. 2. The trajectories of the auxiliary Hamiltonian system (3) form a Lagrangian manifold (LM) in the phase space of the system. Projections of these trajectories onto the $(q, t)$ plane are optimal paths. The Lagrangian manifold in the space $(t, q, p)$ is also shown: note that $p \equiv \partial L / \partial q=\dot{q}-K(q, t)$ is a momentum of the auxiliary system corresponding to the coordinate $q$. Generically the LM can have folds emerging in pairs from the cusp, and it is their projections onto the plane $(q, t)$ that creates the caustics. Caustics and cusp points are the only generic structurally stable singularities of the pattern of extreme paths of $S[q]$ (3) [11]. Since caustics may not [6] be observed in the pattern of optimal paths, it is particularly interesting to investigate the distribution $p_{h}$ near a cusp point $\left(q_{\mathrm{c}}, t_{\mathrm{c}}\right)$. If the final point $\left(q_{f}, t_{f}\right)$ coincides with $\left(q_{\mathrm{c}}, t_{\mathrm{c}}\right)$, then the smallest eigenvalue $\lambda_{\mathbf{0}}=0$ [4] and Eq. (8) does not apply; in particular, $\sigma$ diverges for $\lambda_{0}=0$.

At the cusp point it is necessary [4] to keep in the expansion of the action $s(7)$ the higher-order terms in the amplitude $a_{0}$ of the "soft mode" $\psi_{0}(t)$. Detailed analysis shows $[8]$ that, when $\left(q_{f}, t_{f}\right)$ approaches $\left(q_{c}, t_{c}\right)$, fluctuations 
about the optimal path become strongly non-Gaussian. The characteristic width of the distribution, $\sim D^{1 / 4}$, is determined by the soft mode $\psi_{0}(t)$. The measured and calculated evolutions of the distribution with $t-t_{c}$ are in good agreement: see Fig. 3.

\section{CONCLUSION}

In conclusion, we have used general topological arguments, and the concept of the prehistory probability distribution, to analyze singular features of the pattern of optimal fluctuational paths in systems away from thermal equilibrium. We have described theoretically, and observed, critical broadening of the distribution of paths arriving in the vicinity of a cusp point.

\section{REFERENCES}

1. L. Onsager and S. Machlup, Phys. Rev. 91, 1505, 1512 (1953).

2. See M. Marder, Phys. Rev. Lett. 74, 4547 (1995) and references therein.

3. R. Graham, in Noise in Nonlinear Dynamical Systems, edited by F. Moss and P. V. E. McClintock (Cambridge University, Cambridge, 1989), vol. 1, p. 225.

4. M.V. Berry, Adv. Phys. 25, 1 (1976); L.S. Schulman, Techniques and applications of path integration (Wiley, New York, 1981).

5. H.R. Jauslin, J. Stat. Phys. 42, 573 (1986); Physica 144A, 179 (1987); M.V. Day, Stochastics 20, 121 (1987); Ann. Prob. 20, 1385 (1992); V.A. Chinarov, M.I. Dykman and V.N. Smelyanskiy, Phys. Rev. E 47, 2448 (1993); R.S. Maier and D.L. Stein, Phys. Rev. Lett. 71, 1783 (1993); Phys. Rev. E 48, 931 (1993); S.J.B. Einchcomb and A.J. McKane, Phys. Rev. E 51, 2974 (1995).

6. M.I. Dykman, M.M. Millonas, and V.N. Smelyanskiy, Phys. Lett. A 19553 (1994); R.S. Maier and D.L. Stein, J. Stat. Phys. 83, 291 (1996); V.N. Smelyanskiy, M.I. Dykman, and R.S. Maier, Phys. Rev. E 55, 2369 (1997).

7. M.I. Dykman, P.V.E. McClintock, V.N. Smelyanskiy, N.D. Stein, and N.G. Stocks, Phys. Rev. Lett. 68, 2718 (1992); J. Gómez-Ordóñez, J.M. Casado, and M. Morillo, Phys. Rev. E 54, 2125 (1996); M. Morillo, J.M. Casado, and J. Gómez-Ordóñez, Phys. Rev. E 55, 1521 (1997); B. Vugmeister, J. Botina, and H. Rabitz, Phys. Rev. E 55, 5338 (1997).

8. M.I. Dykman, D.G. Luchinskiy, P.V.E. McClintock and V.N. Smelyanskiy, Phys. Rev. Lett. 77, 5229 (1996).

9. M.I. Freidlin and A.D.Wentzell, Random Perturbations in Dynamical Systems (Springer Verlag, New York, 1984).

10. P.V.E. McClintock and F. Moss in ref. [3], vol. 3, p. 243.

11. H. Whitney, Ann. Math. 62, 374 (1955); V. I. Arnold, Catastrophe Theory (Springer-Verlag, New-York, 1984). 